\title{
The vitamin D analogue paricalcitol attenuates hepatic ischemia/reperfusion injury through down-regulation of Toll-like receptor 4 signaling in rats
}

Min Sung Kim ${ }^{1}$, Soyoung Lee ${ }^{2}$, Namhee Jung ${ }^{3}$, Kiho Lee ${ }^{4}$, Jinwoo Choi ${ }^{3}$, Sang-Hoon Kim³, Jinhyun Jun ${ }^{3}$, Won-Mee Lee ${ }^{3}$, Yeonsoo Chang ${ }^{1}$, Donghee Kim ${ }^{1}$

\author{
${ }^{1}$ Department of Surgery, Eulji General Hospital, Eulji University School of Medicine, \\ Seoul, Korea \\ ${ }^{2}$ Department of Nephrology, Eulji General Hospital, Eulji University School \\ of Medicine, Seoul, Korea \\ ${ }^{3}$ Eulji Medi-Bio Research Institute, Eulji University School of Medicine, Seoul, Korea \\ ${ }^{4}$ Department of Molecular Biology, Eulji University School of Medicine, Daecheon, \\ Korea
}

Submitted: 15 July 2015

Accepted: 9 September 2015

Arch Med Sci 2017; 13, 2: 459-469

DOI: https://doi.org/10.5114/aoms.2016.60650

Copyright (c) 2016 Termedia \& Banach

\begin{abstract}
Introduction: Recent studies have revealed that vitamin D and its synthetic analogues have a protective effect on experimental ischemia/reperfusion $(I / R)$ models in several organs, but little is known about its effect on the liver. The aim of this study was to evaluate the beneficial effects of vitamin $D$ in a model of liver I/R in rats, focusing on Toll-like receptor (TLR) 4 signaling, which has been shown to be involved in I/R injury.

Material and methods: Twenty-four male Wistar rats were randomized into four groups: Saline + Sham, Saline + I/R, Paricalcitol + Sham, and Paricalcitol + I/R. A synthetic vitamin $D_{2}$ analogue, paricalcitol, was intraperitoneally injected $24 \mathrm{~h}$ prior to surgery. The animals were subjected to $60 \mathrm{~min}$ of partial warm ischemia (70\%), followed by reperfusion for $6 \mathrm{~h}$ on the same day. The ischemic lobe of the liver and blood were collected for molecular biochemical analyses.

Results: Liver damage following I/R was diminished by pretreatment with paricalcitol. Pretreatment with paricalcitol decreased the levels of pro-in-

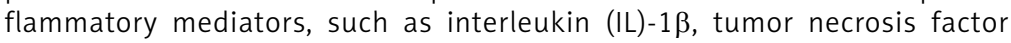
(TNF)- $\alpha$, and macrophage migration inhibitory factor (MIF), in both plasma and liver tissue. In addition, pretreatment with paricalcitol markedly down-regulated the expression of TLR4, HMGB1, TNF- $\alpha$ and NF- $\mathrm{KB}$.

Conclusions: The vitamin $D$ analogue paricalcitol attenuates hepatic I/R injury through down-regulation of the TLR4 signaling pathway and might be considered to be a potential nutritional therapeutic agent against I/R injury in the liver.
\end{abstract}

Key words: hepatic ischemia/reperfusion injury, Toll-like receptor 4 , vitamin D.

\section{Introduction}

Hepatic ischemia/reperfusion (I/R) injury is a pathophysiologic phenomenon in which transient ischemic liver damage is increased despite the resumption of blood flow [1]. Hepatic I/R injury is encountered in var-

\author{
Corresponding author: \\ Donghee Kim MD, PhD \\ Department of Surgery \\ Eulji General Hospital \\ Eulji University School \\ of Medicine \\ 14 Hangeulbiseok-gil \\ Nowon-gu, \\ 01830 Seoul, Korea \\ Phone: 821089214166 \\ E-mail: kdh2109@eulji.ac.kr
}


ious clinical settings, such as hemorrhagic shock with fluid resuscitation, elective liver resection and liver transplantation [2-4]. The mechanisms involved in hepatic $\mathrm{I} / \mathrm{R}$ injury range from initial hypoxic cellular damage to a cascade of deleterious immunologic alterations by pro-inflammatory mediators $[5,6]$. These mediators then recruit monocytes, neutrophils, and $T$ cells into the liver to induce an innate inflammatory response. It has been demonstrated that Toll-like receptors (TLRs), especially TLR4, act as modulators of hepatic I/R injury by inducing an overwhelming innate inflammatory response through macrophages, neutrophils, and the complement pathway [7-9]. Therefore, it is worthwhile to investigate novel agents that can alleviate hepatic I/R injury, especially those that down-regulate TLR4 signaling.

Vitamin $D$ has diverse effects on the immune system, beyond its important role in calcium and phosphorus metabolism [10]. Vitamin D, and particularly its active form, 1,25-dihydroxyvitamin $\mathrm{D}_{3}$ $\left(1,25(\mathrm{OH})_{2} \mathrm{D}_{3}\right)$, has been reported to be protective in several experimental models of inflammatory responses, such as shock and sepsis $[11,12]$. Vitamin $D_{3}$ has also been reported to protect tissue from ischemic injuries, such as myocardial and cerebral ischemia $[13,14]$. A distinct association between vitamin D and TLR4 signaling has been demonstrated. Recently, some investigators have shown that paricalcitol, a synthetic vitamin $D_{2}$ analogue, is renoprotective in experimental models of renal I/R injury [15-17]. Collectively, this evidence highlights that vitamin $D$ and its synthetic analogues play a protective role in various experimental I/R models. However, little is known about its role in hepatic I/R injury. Recently Seif et al. demonstrated that oral vitamin $D$ (cholecalciferol) ameliorated hepatic I/R injury in rats [18]. They suggested that oral vitamin $D$ reduced the levels of pro-inflammatory cytokines through the process attenuating the oxidative stress and apoptotic pathway.

Based on the aforementioned data, we hypothesized that pretreatment with a synthetic vitamin $D_{2}$ analogue, paricalcitol, may attenuate $I / R$ injury through down-regulation of TLR4 signaling in a hepatic model. Therefore, the aim of this study was to evaluate the beneficial effect of paricalcitol in a model of liver I/R in rats, focusing on TLR4 signaling, which has been shown to be involved in $I / R$ injury.

\section{Material and methods}

\section{Animals}

Male Wistar rats weighing 200-250 g at 7 weeks of age were purchased from Orient-Bio Laboratory Animal Research Center Co., Ltd. (Seongnam-si,
Gyeonggi-do, Korea). All of the animal procedures were performed in accordance with the guidelines set out by the Animal Care and Use Committee of Eulji University School of Medicine (EUIACUC 1306).

\section{Liver ischemia}

We used an I/R protocol involving a nonlethal model of partial (70\%) hepatic warm ischemia, which was described in our previous study [19]. A reproducible level of hepatic damage was observed using $60 \mathrm{~min}$ of ischemia and was thus used for our study. A midline abdominal incision was used to access the whole liver. Using an operating microscope, the liver hilum was dissected. All the structures in the portal triad to the left and the median liver lobes were occluded with a microvascular clamp (Jeongdo B\&P, Seoul, Korea) for $60 \mathrm{~min}$, and then reperfusion was initiated by removal of the clamp. After application of the clamp, the abdomen was covered with a sterile plastic wrap to minimize evaporative loss. Throughout the ischemic interval, evidence of ischemia was confirmed by visualizing the blanching of the ischemic lobes. The clamp was then removed and gross evidence of reperfusion, based on immediate color change, was confirmed before closing the abdomen. Sham animals underwent anesthesia, laparotomy, and exposure of the portal triad without hepatic ischemia.

\section{Experimental design and drugs}

Twenty-four male Wistar rats were randomized into four groups. All of the experimental rats were fed and housed at Eulji Medical University, following the above-mentioned guidelines. The rats were divided into the following groups: Saline + Sham group $(n=6)$, intraperitoneal (ip) injection of saline $24 \mathrm{~h}$ prior to sham laparotomy; Paricalcitol + Sham group $(n=6)$, an equivalent volume of paricalcitol (Zemplar; Abbott Laboratories, Abbott Park, IL, USA) $(20 \mu \mathrm{g} / \mathrm{kg}$ ip) injection $24 \mathrm{~h}$ prior to sham laparotomy; Saline + I/R group $(n=6)$, pretreatment with saline (ip) injection $24 \mathrm{~h}$ prior to hepatic I/R; and Paricalcitol + I/R group $(n=6)$, pretreatment with paricalcitol (20 $\mathrm{gg} / \mathrm{kg}$ ip) injection $24 \mathrm{~h}$ prior to hepatic I/R. The proper dosage and injection time of paricalcitol prior to surgery were determined by a series of preliminary examinations (data not shown).

\section{Peripheral blood and liver tissue collection}

At a predetermined time point after reperfusion $(6 \mathrm{~h})$, the rats were anesthetized with inhaled methoxyflurane, plasma and liver samples were collected, and then rats were sacrificed by exsanguination. Blood samples were collected 
from the inferior vena cava via the previous midline laparotomy with a sterile heparinized syringe containing $50 \mu \mathrm{l}$ of heparin (100 USP U/ml). The blood samples were centrifuged and plasma was collected and stored at $-80^{\circ} \mathrm{C}$ until use. Portions of the ischemic and non-ischemic liver lobes were fixed in $10 \%$ buffered formalin, embedded in paraffin, and used for hematoxylin and eosin $(\mathrm{H}+\mathrm{E})$ staining and immunohistochemical studies. Other portions of the ischemic and non-ischemic liver lobes were snap-frozen in liquid nitrogen and stored at $-80^{\circ} \mathrm{C}$ until use in enzyme-linked immunosorbent assays (ELISA) and western blotting analysis.

\section{Biochemical analysis}

To assess hepatic function and cellular injury following liver ischemia, the plasma alanine aminotransferase (ALT) levels were measured using the Opera Clinical Chemistry System (Bayer, Munich, Germany). The calcium levels were measured using an IDEXX VetTest Chemistry Analyzer (IDEXX Laboratories, Inc., Westbrook, ME, USA).

\section{Histopathological examination}

The $10 \%$ formalin-fixed liver samples were embedded in paraffin and cut into $5-\mu \mathrm{m}$ thick sections. Tissues were stained with $\mathrm{H}+\mathrm{E}$, and slides were assessed for inflammation and tissue injury using an Olympus microscope (Olympus Optical Co., Tokyo, Japan) at 200× magnification. The percentage of necrotic area was estimated by random evaluation of three low-power fields (100x) per each $\mathrm{H}+\mathrm{E}$ section. A single pathologist, blinded to the experimental procedure, examined the histopathology of the hepatic tissue sections.

\section{Immunohistochemical staining}

Paraffin-embedded sections $(5 \mu \mathrm{m})$ were blocked with $5 \%$ normal goat serum (Chemicon International, Temecula, CA, USA) for $30 \mathrm{~min}$, and then stained with primary antibodies. The dilutions of primary antibodies used in the present study were 1 : 200 for NF- $\kappa B$ (polyclonal rabbit anti-NF-кB p65; Abcam, Cambridge, MA, USA), 1 : 400 for TLR4 (polyclonal rabbit anti-TLR4; Abcam), and 1 : 1,000 for VDR (polyclonal rabbit anti-VDR; Abcam). Positive immunostaining was detected by treatment with a mixture of 3,3'-diaminobenzidine (Sigma, St. Louis, MO, USA), 0.05 M Tris- $\mathrm{HCl}$ buffer, and $5 \% \mathrm{H}_{2} \mathrm{O}_{2}$. The tissue sections were counterstained with hematoxylin and were then dehydrated using a graded ethanol series. The levels of TLR4, NF-KB, and VDR expression were determined by blind unbiased reading (the pathologist was not aware of which treatment the animal received).

\section{ELISA for inflammatory cytokines in plasma and liver tissue}

The plasma levels of tumor necrosis factor $\alpha$ (TNF- $\alpha$ ), interleukin (IL)-1 $\beta$, and macrophage migration inhibitory factor (MIF) were determined in a 96-well Nunc-Immuno microplate (VWR Scientific, Chicago, IL, USA) by ELISA, according to the manufacturer's instructions (Medical \& Biological Laboratories Co., Ltd.; Biosource International, Camarillo, CA; R\&D Systems, Inc., Minneapolis, MN, USA). The capture antibody was a polyclonal antimouse TNF- $\alpha$, IL-1 $\beta$, and MIF-specific goat IgG (R\&D Systems), and the detection antibody was a biotinylated polyclonal anti-mouse TNF- $\alpha$, IL-6, or MIF-specific goat IgG (R\&D Systems). All of the plasma samples were tested in duplicate. The minimal detectable protein concentration was $20 \mathrm{pg} / \mathrm{ml}$, and the range of detection for each cytokine was $20-5000 \mathrm{pg} / \mathrm{ml}$. The total protein concentration of each sample was measured using a bicinchoninic assay kit (Pierce Chemical Co. Rockford, IL, USA), and the samples were dispensed for ELISA assays. The cytokine concentration was calculated in pg of total protein per $\mathrm{mL}$ of tissue, as described previously.

\section{SDS-PAGE and western blot analysis}

To analyze the severity of inflammation in the ischemic lobe, frozen liver tissues were lysed in ice-cold RIPA buffer supplemented with protease inhibitors (Complete; Roche Applied Science, Indianapolis, IN, USA). Whole cell lysates were obtained by centrifugation at 4,000 $\mathrm{g}$ for $10 \mathrm{~min}$ at $4^{\circ} \mathrm{C}$. After the protein concentration was determined using the BCA protein assay, equal volumes of lysate were fractionated by SDS-PAGE and transferred to nitrocellulose membranes for $2 \mathrm{~h}$ at $250 \mathrm{~mA}$ in the presence of transfer buffer. The transferred membrane was blocked for $1 \mathrm{~h}$ at room temperature with $5 \%$ milk in TBS-T buffer and incubated with a primary antibody to TLR4 (1 : 1000; Abcam), high mobility group box 1 (HMGB1, 1 : 1000; Cell Signaling Technology, Danvers, MA, USA), p65 (1 : 1000; Cell Signaling Technology), TNF- $\alpha$ (1 : 1000; Cell Signaling Technology), and vitamin D receptor (VDR, $1: 1000 ;$ Abcam) for $12 \mathrm{~h}$ at $4^{\circ} \mathrm{C}$. The blots were incubated at room temperature for $1 \mathrm{~h}$ with a horseradish peroxidase-conjugated secondary antibody against the primary antibody. After extensive washing with TBS-T buffer, the membranes were developed using the Super Signal West Pico Chemiluminescent Kit (Pierce Chemical Co.) and exposed to film.

\section{Statistical analysis}

Data were analyzed using the statistical program SPSS (Statistical Package for the Social Sci- 
ences, Version 18 for Windows, SPSS Inc., Chicago, IL, USA). The results are expressed as the mean \pm standard error of the mean. Differences between groups were evaluated for significance using oneway ANOVA combined with Bonferroni's post hoc test. A $p$-value less than 0.05 was considered statistically significant.

\section{Results}

\section{Pretreatment with paricalcitol attenuates hepatocellular injury after I/R}

To determine whether paricalcitol attenuated liver parenchymal injury after I/R, paricalcitol was administered to rats that were subjected to I/R. The rats were injected with paricalcitol $(20 \mu \mathrm{g} / \mathrm{kg}$ per rat) or an equivalent volume of saline $24 \mathrm{~h}$ prior to ischemia or sham laparotomy. We examined the plasma alanine aminotransferase (ALT) levels and histopathologic features of ischemic liver sections to assess hepatocellular damage. As shown in Figure $1 \mathrm{~A}$, plasma ALT levels showed no significant difference between the Saline + Sham group and the Paricalcitol + Sham group $(59.83 \pm 2.06 \mathrm{U} / \mathrm{l}$ vs. $59.33 \pm 1.52 \mathrm{U} / \mathrm{l}, p=0.81$ ). The paricalcitol itself had no influence on the plasma ALT levels. After hepatic I/R, the plasma ALT levels were significantly elevated in the Saline + I/R group compared with the Saline + Sham group $(1919.5 \pm 219.69 \mathrm{U} / \mathrm{l}$ vs. $59.83 \pm 2.06 \mathrm{U} / \mathrm{l}, p=0.004)$. The plasma ALT levels in the Paricalcitol + I/R group were significantly lower than those in the Saline $+1 / R$ group $(507.83 \pm 90.21 \mathrm{U} / \mathrm{l}$ vs. $1919.5 \pm 219.69 \mathrm{U} / \mathrm{l}$, $p=0.004)$. Pretreatment with paricalcitol attenuated the elevation of the plasma ALT levels in rats after hepatic I/R. The plasma calcium levels were not significantly different between the four groups (Figure 1 B). The plasma calcium levels were not affected by paricalcitol. As expected from the plasma ALT levels, no morphological alterations were observed in the liver sections from the Saline + Sham and the Paricalcitol + Sham groups (Figures 2 A, B), whereas the liver sections from the Saline + I/R group (Figure $2 \mathrm{C}$ ) showed periportal inflammation, sinusoidal destruction, and hepatocellular necrosis, as well as inflammatory cell infiltration in extensive portions. However, these morphological changes were decreased in the Paricalcitol + I/R group (Figure 2 D)

\section{Pretreatment with paricalcitol decreases the production of pro-inflammatory mediators after I/R}

To determine whether paricalcitol alters the production of pro-inflammatory mediators, we measured the levels of IL-1 $\beta$, TNF- $\alpha$, and MIF in plasma and liver tissue after I/R (Figure 3). Compared with the Saline + Sham group, the expression of IL- $1 \beta$, TNF- $\alpha$, and MIF was significantly increased in plasma and ischemic liver tissues of the Saline $+1 / R$ group. However, the expression of IL-1 $\beta$, TNF- $\alpha$, and MIF was significantly decreased in plasma and the ischemic liver tissues of the Paricalcitol + I/R group compared with the Saline + I/R group. In the plasma, the IL-1 $\beta$ levels were
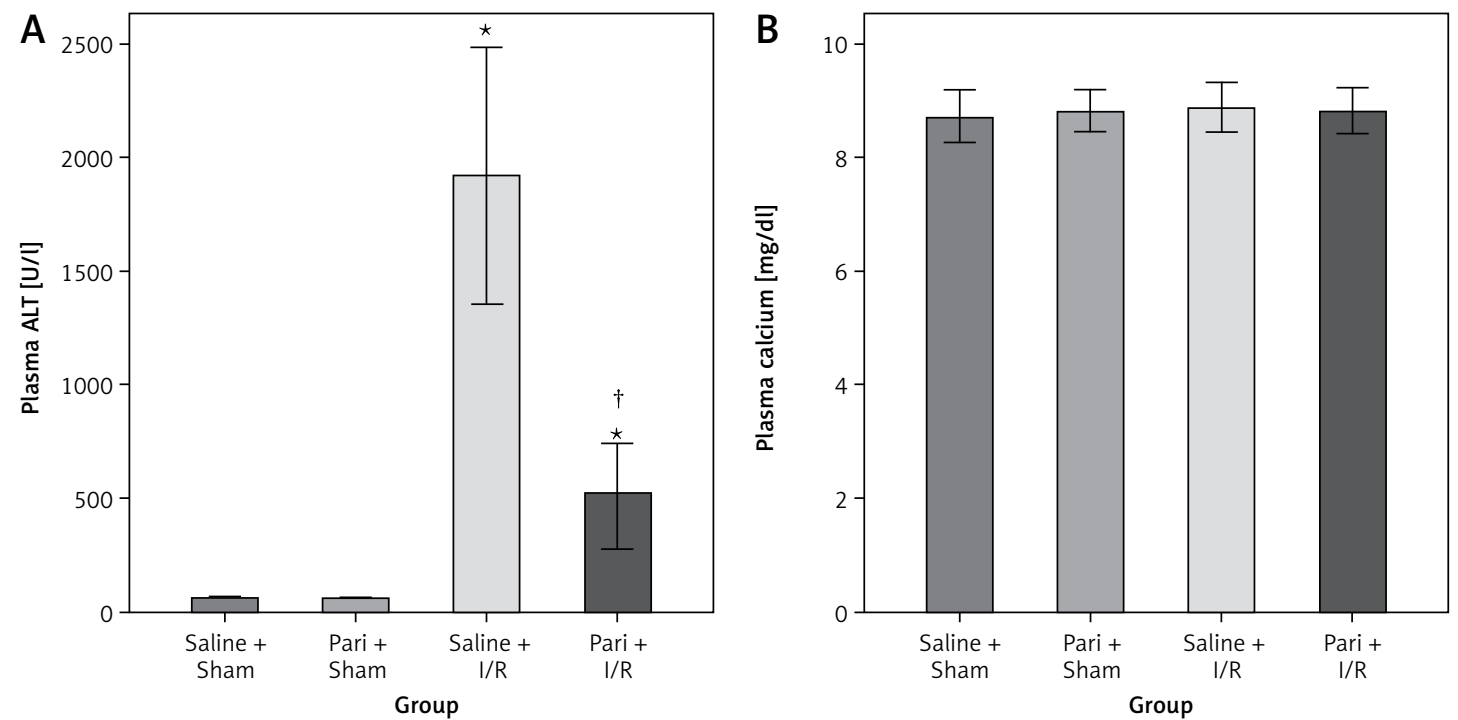

Figure 1. Effects of paricalcitol pretreatment on serum ALT and calcium levels in the rats that underwent hepatic I/R injury. A - The serum ALT levels were analyzed as a measure of hepatocellular injury. Pretreatment with paricalcitol induced a significant, marked attenuation of elevated serum ALT levels $6 \mathrm{~h}$ after hepatic I/R. B - Pretreatment with paricalcitol did not change the serum calcium levels at $6 \mathrm{~h}$ after hepatic I/R

The values shown are expressed as the mean \pm S.E.M., $n=6$ rats per group. ${ }^{*} p<0.05$, compared with the Saline + Sham group, ${ }^{t} p<0.05$, compared with the Saline $+I / R$ group. Error bars represent the standard deviation. ALT - alanine aminotransferase, $I / R$ - ischemia reperfusion. 

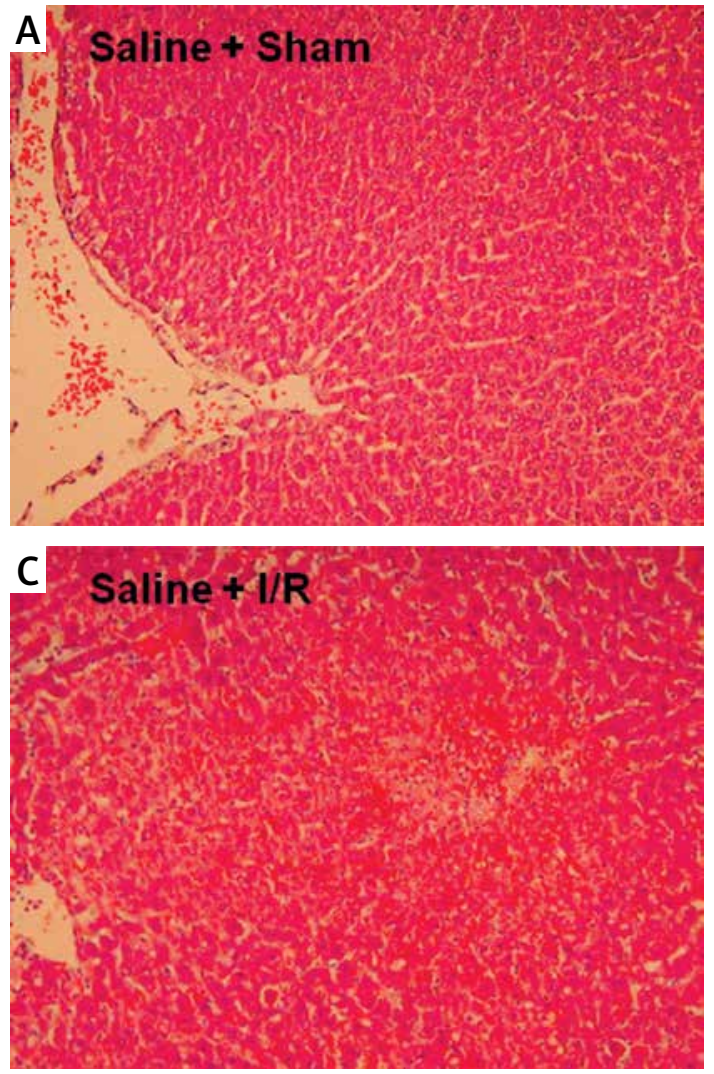

E

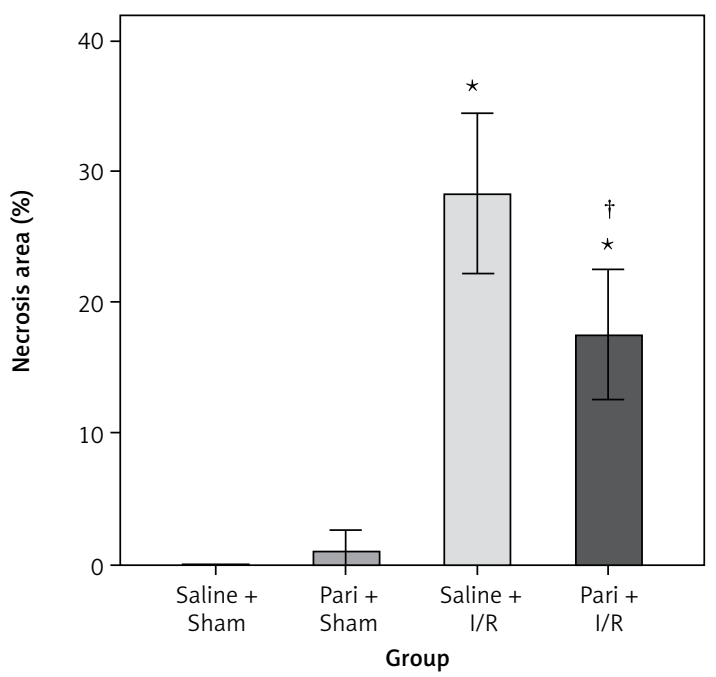

significantly increased in the Saline + I/R group compared with the Saline + Sham group (123.52 $\pm 2.34 \mathrm{pg} / \mathrm{ml}$ vs. $11.48 \pm 0.85 \mathrm{pg} / \mathrm{ml}, p=0.004$, Figure $3 \mathrm{~A}$ ), and this elevated expression was markedly decreased in the Paricalcitol + I/R group compared with the Saline + I/R group $(92.17 \pm 9.89 \mathrm{pg} /$ $\mathrm{ml}$ vs. $123.52 \pm 2.34 \mathrm{pg} / \mathrm{ml}, p=0.004$, Figure $3 \mathrm{~A}$ ). In addition, the expression of TNF- $\alpha$ and MIF was increased in the Saline + I/R group compared with the Saline + Sham group $(p<0.05$ versus the Saline + Sham group), and the elevation of expression was significantly decreased in the Paricalcitol
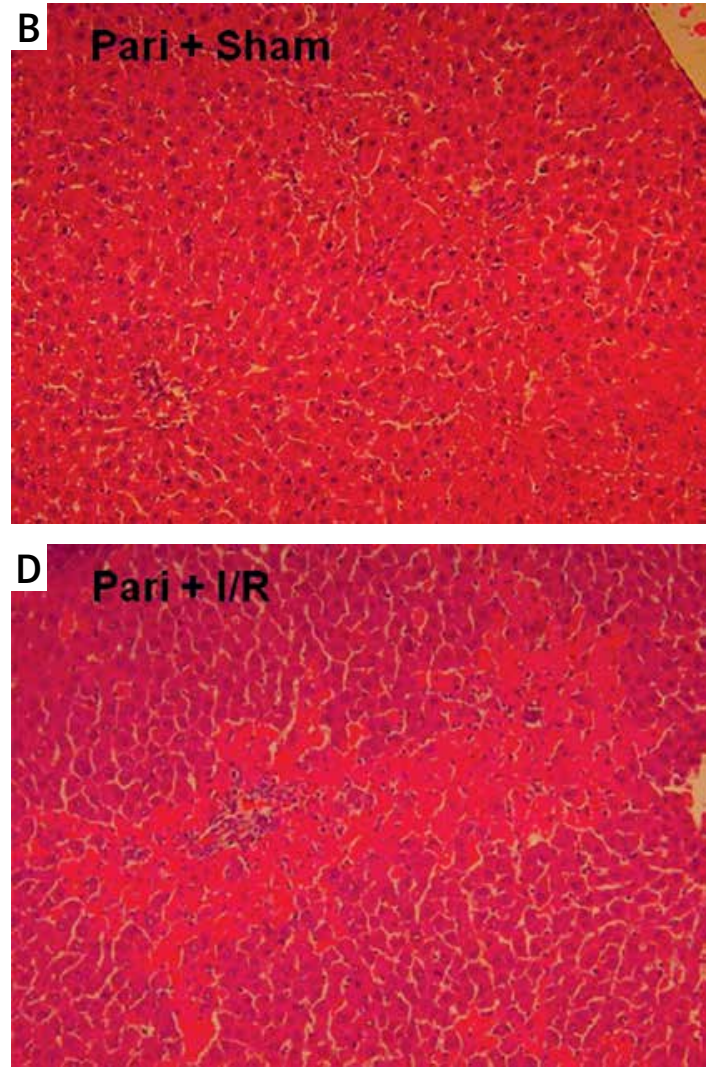

Figure 2. Effects of paricalcitol pretreatment on the histopathological features of liver sections in rats that underwent hepatic I/R injury. Representative images were chosen from each experimental group $(H+E, 200 x)$. A - In the Saline + Sham group, the lobular architecture and cell structure were normal. B - In the Paricalcitol + Sham group, some sinusoidal breakdown and cell swelling can be seen. $\mathbf{C}$ - In the Saline + I/R group, the hepatic lobules show large foci of coagulation necrosis and adjacent ballooning degeneration with extensive inflammation and inflammatory cell infiltration. D - In the Paricalcitol + I/R group, the hepatic lobules show some coagulation necrosis and adjacent ballooning degeneration with mild inflammation and inflammatory cell infiltration compared to the Saline + I/R group. E - The percentages of necrotic area were analyzed as a measure of hepatocellular injury in four groups. Pretreatment with paricalcitol resulted in a significantly smaller hepatic necrotic area in the Paricalcitol + I/R group, compared with the Saline + I/R group

${ }^{*} p<0.05$, compared with the Saline + Sham group, ${ }^{\dagger} p<0.05$, compared with the Saline $+1 / R$ group. Error bars represent the standard deviation. $I / R$ - ischemia reperfusion.

$+1 / R$ group compared with the Saline $+1 / R$ group $(p<0.05$ vs. the Saline $+1 / R$ group, Figures 3 C, E). In the liver tissue, the IL-1 $\beta$, TNF- $\alpha$, and MIF levels were also significantly increased in the Saline + I/R group compared with the Saline + Sham group ( $p<0.05$ vs. the Saline + Sham group) and the elevation of expression was significantly decreased in the Paricalcitol $+1 / R$ group $(p<0.05$ vs. the Saline $+\mathrm{l} / \mathrm{R}$ group, Figures $3 \mathrm{~B}, \mathrm{D}, \mathrm{F})$.

Although the data are not shown in Figure 3, the expression of IL-1 $\beta$, TNF- $\alpha$, and MIF was increased in plasma and ischemic liver tissues of 
A

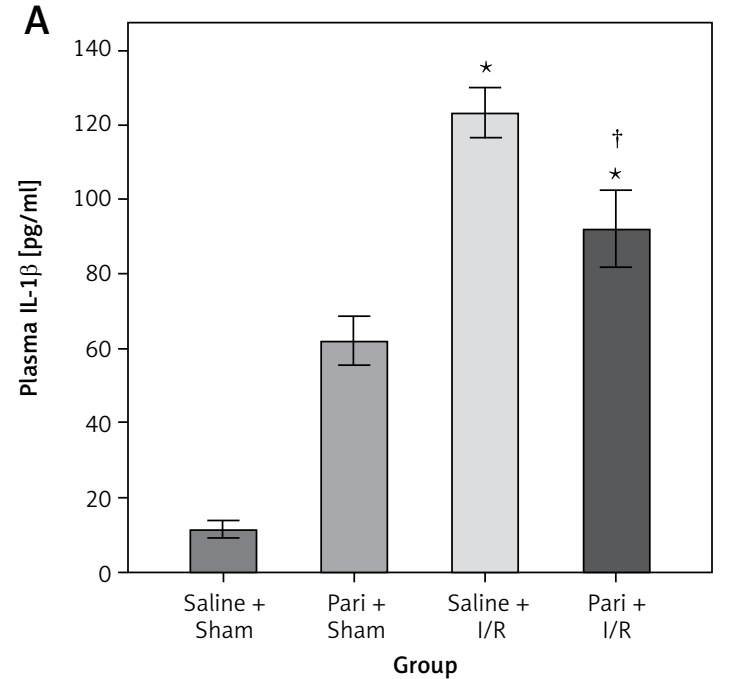

C

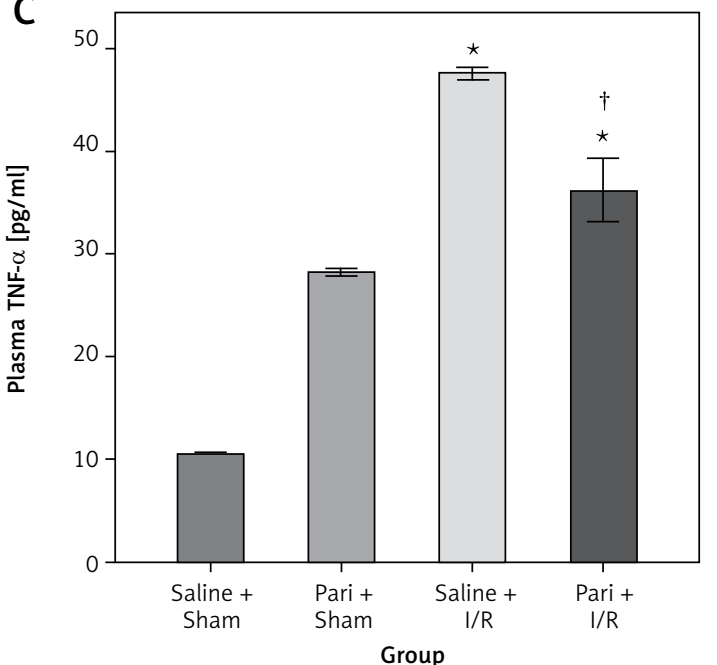

$E$

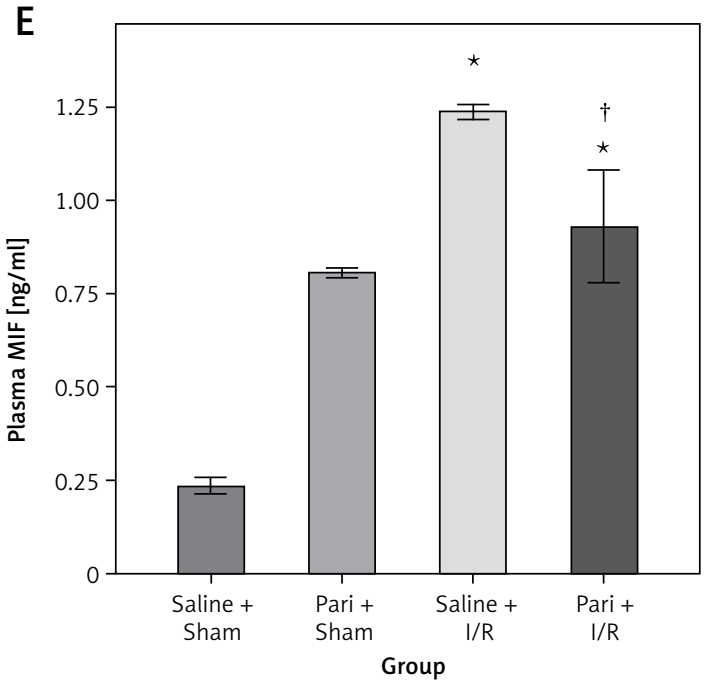

B

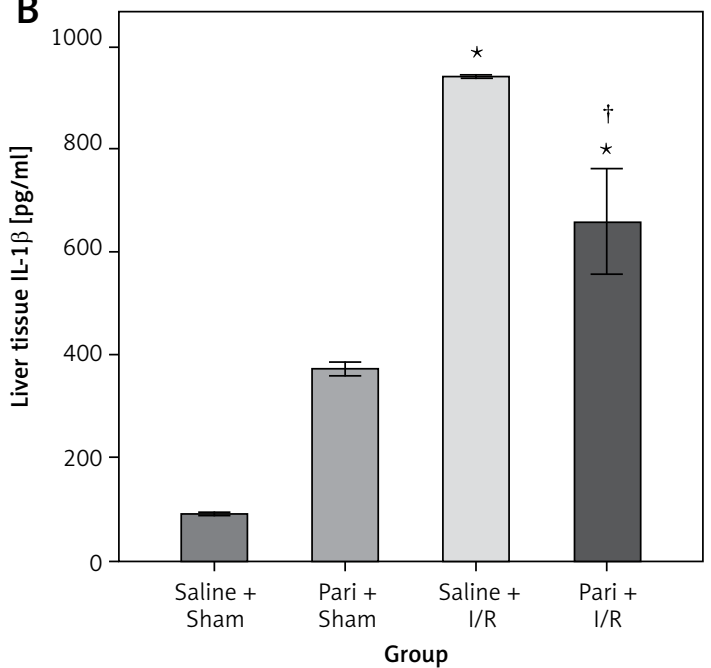

D

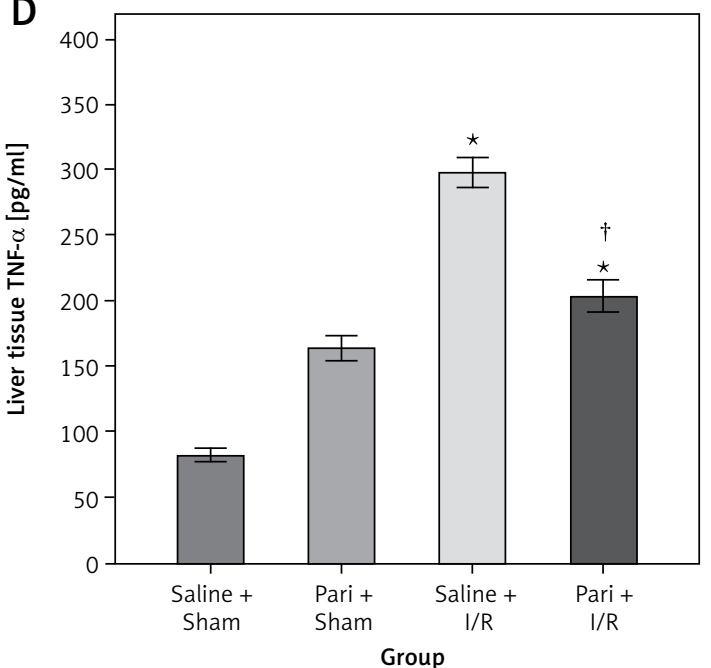

$\mathrm{F}$

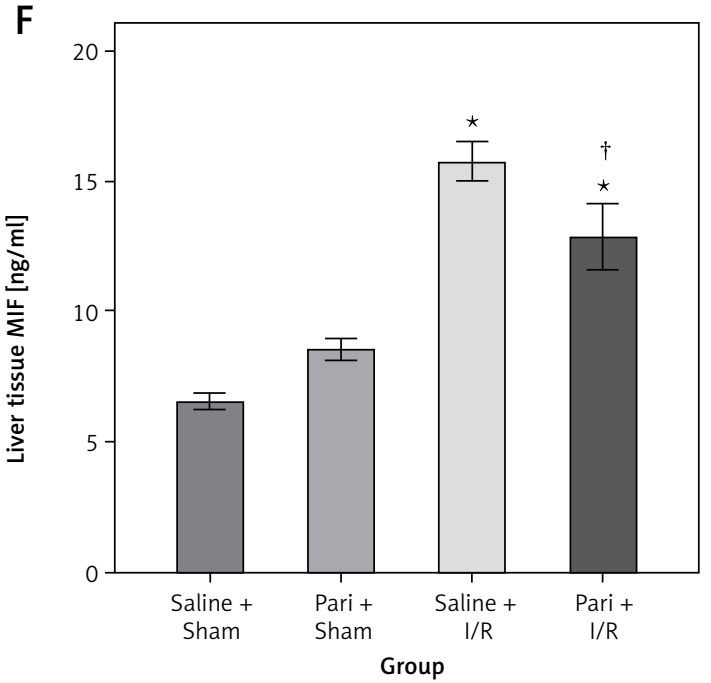

Figure 3. Effects of paricalcitol pretreatment on proinflammatory mediators in the serum and liver tissue of rats that underwent hepatic I/R injury. The ELISA results show that IL-1 $\beta$ (A and B), TNF- $\alpha$ ( $C$ and $\mathbf{D}$ ), and MIF (E and F) are significantly elevated in the serum and ischemic liver tissue of the Saline $+I / R$ group. In contrast, these mediators are down-regulated in the Paricalcitol + I/R group compared to the Saline + I/R group. Pretreatment with paricalcitol significantly reduced proinflammatory mediators, such as IL-1 $\beta$, TNF- $\alpha$, and MIF, in the serum and ischemic liver tissue

The values shown are expressed as the mean \pm S.E.M., $n=6$ rats per group. ${ }^{*} p<0.05$, compared to the Saline + Sham group, ${ }^{\dagger} p<$ 0.05 , compared to the Saline $+1 / R$ group. Error bars represent the standard deviation. ELISA - enzyme-linked immunosorbent assay, $I / R$ - ischemia reperfusion, IL-1 $\beta$ - interleukin-1 $\beta$, TNF- $\alpha$ - tumor necrosis factor- $\alpha$, MIF - macrophage migration inhibitory factor. 
the Paricalcitol + Sham group, compared with the Saline + Sham group. In the plasma, IL-1 $\beta$ levels were significantly increased in the Paricalcitol + Sham group compared with the Saline + Sham group $(62.08 \pm 2.39 \mathrm{pg} / \mathrm{ml}$ vs. $11.48 \pm 0.85 \mathrm{pg} / \mathrm{ml}$, $p=0.004)$. In addition, the expression of TNF- $\alpha$ and MIF was increased in the Paricalcitol + Sham group compared with the Saline + Sham group (all $p<0.05$ versus the Saline + Sham group). In the liver tissue, IL-1 $\beta$, TNF- $\alpha$, and MIF levels were also significantly increased in the Paricalcitol + Sham group compared with the Saline + Sham group (all $p<0.05$ vs. the Saline + Sham group).

\section{Pretreatment with paricalcitol down- regulates TLR4/NF- $\kappa B$ signaling after I/R}

To determine whether paricalcitol affects TLR4/ NF- $\mathrm{B}$ signaling, we assessed the expression of TLR4, HMGB1, and NF- $\kappa B$ in ischemic liver tissues using western blot analysis (Figure 4) and immunohistochemical staining (Figure 5). Figure 4 shows the outcomes of the western blot analysis of TLR4, HMGB1, and NF- $\kappa B$ expression. After hepatic I/R, the expression of TLR4, HMGB1, and TNF- $\alpha$ was up-regulated in the Saline $+I / R$ group, but pretreatment with paricalcitol markedly attenuated the expression of TLR4, HMGB1, and TNF- $\alpha$ in the Paricalcitol + I/R group. In the analysis of NF- $\kappa B$ activation, the p65 subunit of NF- $\kappa B$ was up-regulated in the Saline + I/R group, but this expression was also down-regulated in the Paricalcitol + I/R group. Figure 5 shows the outcomes of the immunohistochemical analyses of TLR4 and NF- $\kappa B$. The expression of TLR4 and $\mathrm{NF}-\kappa \mathrm{B}$ is shown as dark brown staining. Increased expression of TLR4 near the injured tissues was noted in the Saline + I/R group. In the Paricalcitol + I/R group, the expression of TLR4 was decreased compared to the Saline + I/R group (Figures $5 \mathrm{~A}-\mathrm{D}$ ). Expression of $\mathrm{NF}-\mathrm{\kappa B}$ was similar to that of TLR4 in the liver tissues of each group (Figures $5 \mathrm{E}-\mathrm{H}$ ).

\section{Vitamin D receptor is up-regulated after I/R and pretreatment with paricalcitol attenuates the expression of VDR}

To investigate the possible link between vitamin $D$ receptor (VDR) and hepatic I/ $R$ injury, we assessed the expression of VDR in ischemic liver tissues using western blot analysis and immunohistochemical staining (Figure 6). The expression of VDR was paralleled by the pattern of TLR4 and NF- $\kappa B$ expression in the liver tissues of each group. After hepatic $1 / R$, the expression of VDR was up-regulated in the Saline $+I / R$ group, but the expression of VDR was down-regulated in the Paricalcitol + I/R group.

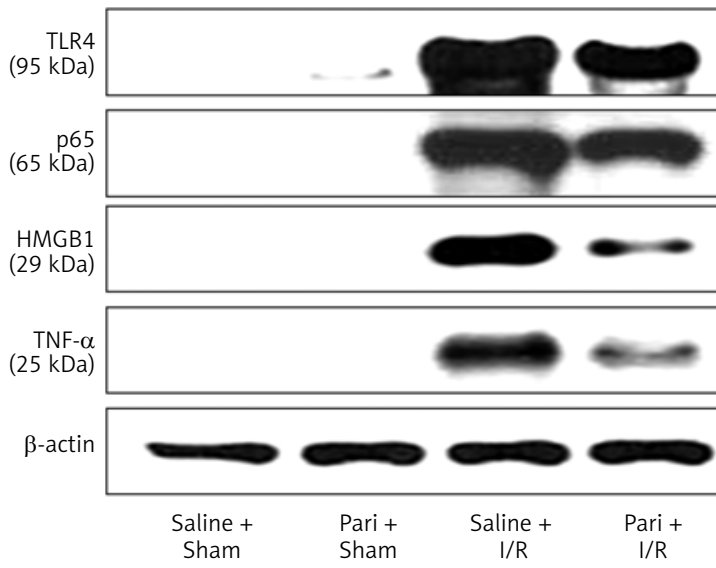

Figure 4. Effects of paricalcitol pretreatment on protein expression of TNF- $\alpha$, HMGB1, p65 NF- $\kappa B$, and TLR4 in the liver tissue of rats that underwent hepatic I/R injury. Western blot analysis shows that pretreatment with paricalcitol significantly attenuated the protein expression of TNF- $\alpha$, HMGB1, p65 NF- $\kappa B$, and TLR4 in ischemic liver tissue. The expression of TNF- $\alpha$, HMGB1, p65 NF- $\kappa \mathrm{B}$, and TLR4 is not observed in the Saline + Sham group. These mediators are up-regulated in the Saline + I/R group. In contrast, the expression of TNF- $\alpha$, HMGB1, p65 NF- $\kappa \mathrm{B}$, and TLR4 is down-regulated in the Paricalcitol + I/R group compared to the Saline + I/R group

The blot shown is representative of 6 experiments with similar results. I/R - ischemia reperfusion, TNF- $\alpha$ - tumor necrosis factor- $\alpha$, HMGB1 - high mobility group box 1 ,

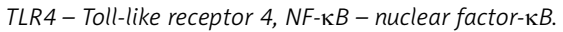

\section{Discussion}

Since Buetler et al. initially reported that TLR4 is essential for the recognition of bacterial lipopolysaccharide (LPS), follow-up studies have shown that TLR4 also plays a role in the activation of inflammatory responses in the setting of acute sterile insults, such as hepatic I/R [20, 21]. The use of nutritional therapeutics for down-regulation of TLR4 signaling has attracted attention for its potential to limit hepatic I/R injury. In this study, we sought to determine whether paricalcitol, a synthetic vitamin D analogue, could block hepatic TLR4-induced inflammation and prevent organ damage after I/R. When male Wistar rats were pre-treated with paricalcitol and subjected to warm hepatic I/R, the plasma biochemical parameters and histopathologic findings of hepatocellular injury showed significant improvement in the pretreated group compared with control counterparts. Additionally, we found that pretreatment with paricalcitol was able to down-regulate the expression of HMGB1/TLR4, and the downstream molecules activated NF- $\mathrm{B}$ p 65 and prevented the secretion of pro-inflammatory mediators, such as IL-1 $\beta$, TNF- $\alpha$, and MIF, in ischemic liver tissues.

Vitamin $D$ is essential for regulating calcium, phosphorus, and bone metabolism in the body 

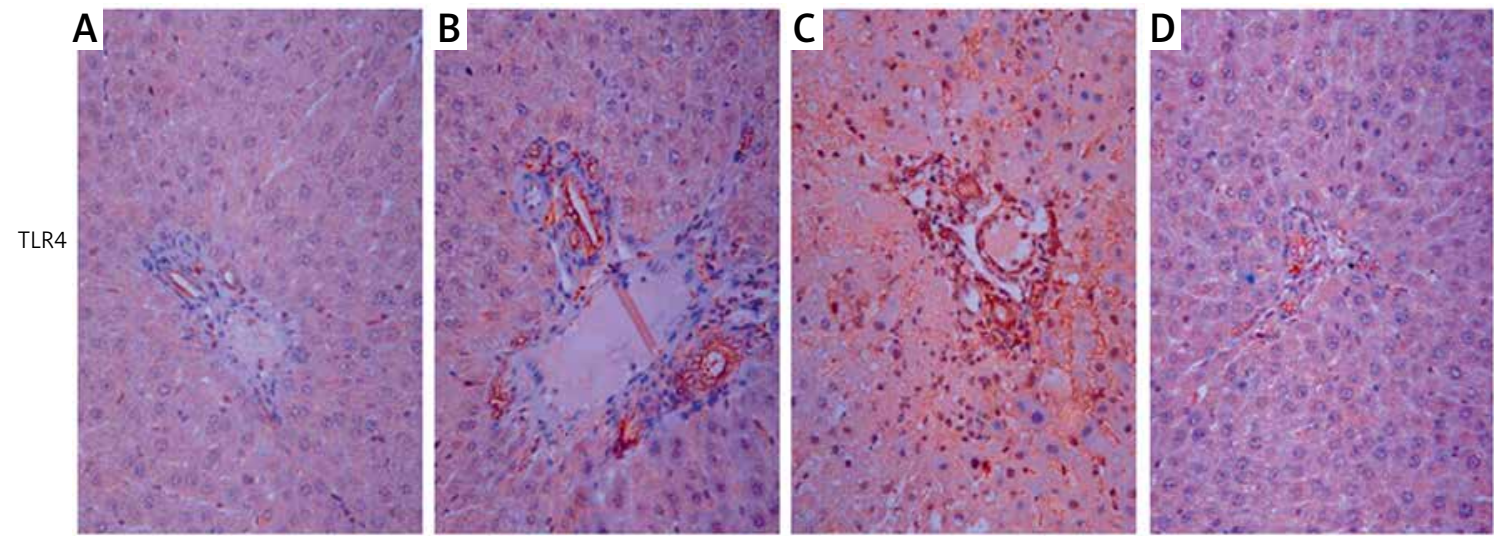

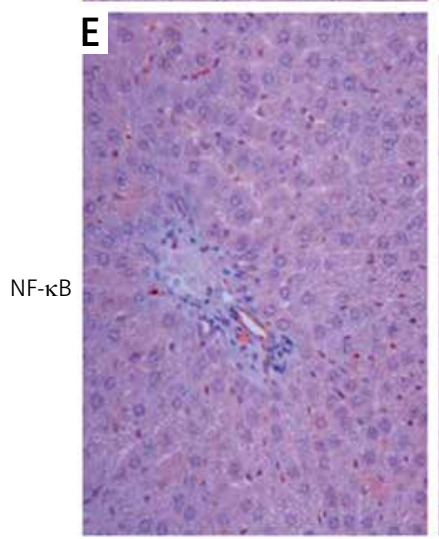

Saline + Sham

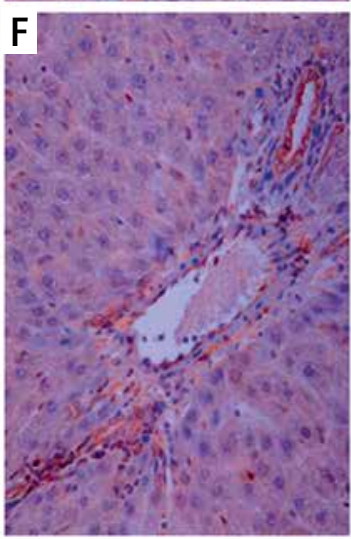

Pari + Sham

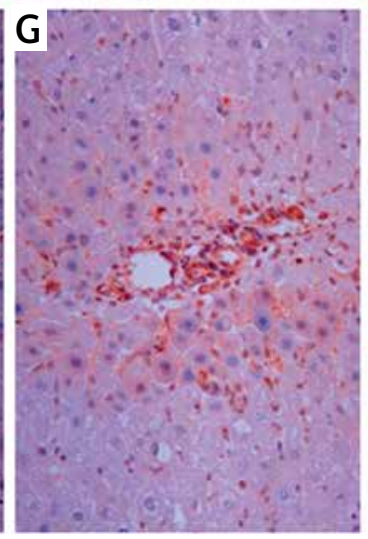

Saline $+\mathrm{I} / \mathrm{R}$

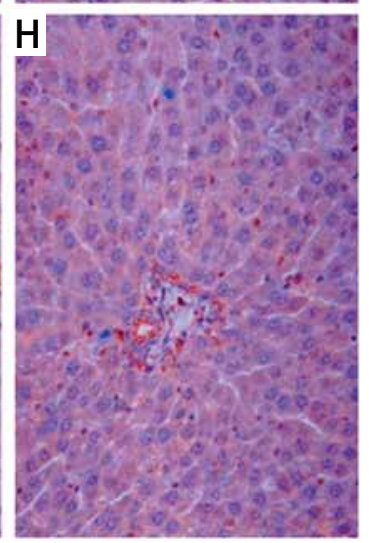

Pari + I/R

Figure 5. Immunohistochemical study of TLR4 and NF-KB in liver tissues (400x). Representative images were chosen from each experimental group. Immunohistochemical analysis shows that pretreatment with paricalcitol significantly reduced the expression of TLR4 and NF-KB in liver tissue. (A-D) Immunohistochemical staining for TLR4 in the liver sections of each group. TLR4 expression is shown by dark brown staining in the cytoplasm or the nucleus of hepatocytes. A - In the Saline + Sham group, TLR4 staining is not observed. B - In the Paricalcitol + Sham group, TLR4 expression is very low or absent. C - In the Saline + I/R group, progressively increasing TLR4 expression is observed near the injured tissues. D - In the Paricalcitol + I/R group, TLR4 expression is attenuated compared to the Saline + I/R group. Immunohistochemical staining for TLR4 reveals decreased TLR4 staining compared with the Saline + I/R group. E-H - Immunohistochemical staining for NF-KB in liver sections adjacent to TLR4 immunohistochemical staining. The expression of NF- $\mathrm{KB}$ is paralleled by the pattern of TLR4 expression in the liver sections of each group

$N F-\kappa B$ expression is also indicated by dark brown staining. I/R - ischemia reperfusion, TLR4-Toll-like receptor 4, NF- $\kappa B-n u c l e a r$ factor- $\mathrm{B}$.

(its "classical" actions) [10, 22]. Beyond its classical actions, vitamin $\mathrm{D}$ also has distinct roles in the control of metabolism, cellular growth, and the immune system ("non-classical" actions) $[10,22]$. The non-classical actions of vitamin D were identified for the first time in 1980 when receptors for active 1,25-dihydroxyvitamin $D_{3}$ $\left(1,25(\mathrm{OH})_{2} \mathrm{D}_{3}\right)$ were detected in various neoplastic cell lines [23]. The spectrum of its non-classical actions was then extended to include acting on cells from the immune system. For many years, it has been considered that the key role of vitamin $D$ in innate immunity was its ability to stimulate the differentiation of precursor monocytes into more mature phagocytic macrophages [24, 25]. However, the confirmation of such a mechanism was only obtained in 2006 when Liu et al. performed DNA array analyses [26]. They reported that TLR activation in human monocytes and macrophages induces up-regulation of VDR and vitamin-D-1 hydroxylase gene expression [26]. On the other hand, Sadeghi et al. demonstrated that vitamin $D_{3}$ led to a decrease of the mRNA and protein expression of TLR2 and TLR4 in human monocytes after lipopolysaccharide stimulation [27]. This resulted in the decreased production of pro-inflammatory mediators, as well as the decreased translocation of NF- $\kappa B$ to the nucleus [27].

Collectively, the aforementioned evidence suggests that vitamin D may play a role in modulating the immune system. Furthermore, there is a novel idea that active 1,25-dihydroxyvitamin $D_{3}$ $\left(1,25(\mathrm{OH})_{2} \mathrm{D}_{3}\right)$ and its analogues might become useful in the prevention or treatment of deleterious immunologic alteration such as I/R injury. So, we hypothesized that pretreatment with parical- 


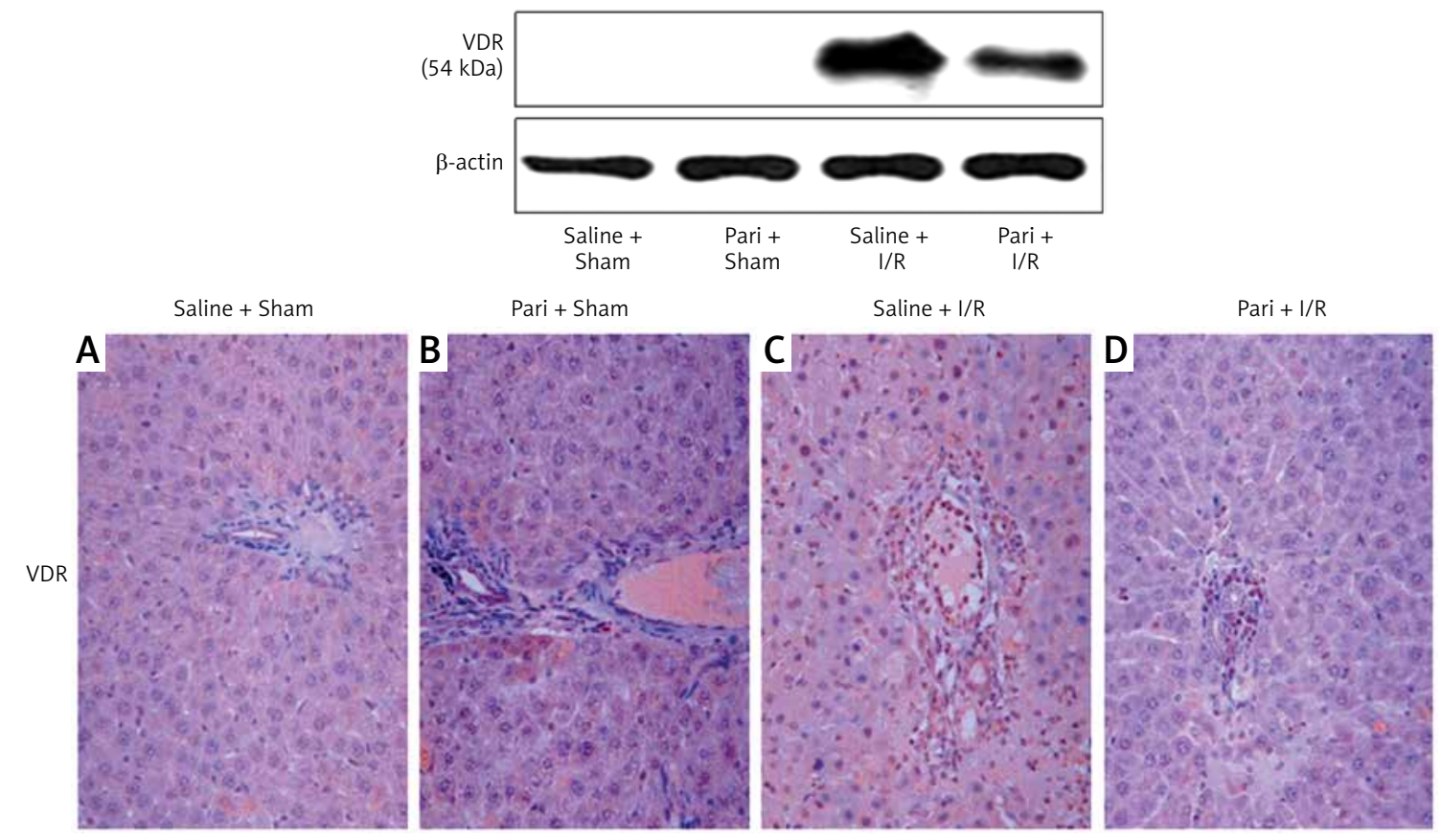

Figure 6. Effects of paricalcitol pretreatment on VDR expression in the liver tissue of rats that underwent hepatic I/R injury. The results of western blot and immunohistochemical analysis show that VDR expression is significantly increased in ischemic liver tissue, but pretreatment with paricalcitol significantly attenuated VDR expression in ischemic liver tissue. The expression of the VDR is paralleled by the pattern of TLR4 and NF-kB expression in the liver sections of each group. Western blotting shows that VDR expression is significantly up-regulated in the Saline + I/R group, but VDR expression is down-regulated in the Paricalcitol + I/R group compared to the Saline + I/R group. (A-D, 400x) Immunohistochemical staining for VDR in the liver sections of each group. VDR expression is shown by dark brown staining in the cytoplasm or nucleus of sinusoidal endothelial, Kupffer, and biliary epithelial cells and hepatocytes. A - In the Saline + Sham group, VDR staining is not observed. B - In the Paricalcitol + Sham group, VDR expression is very low or absent. $C-$ In the Saline + I/R group, progressively increasing VDR expression is observed near the injured tissues. D - In the Paricalcitol + I/R group, VDR expression is attenuated compared to the Saline + I/R group

The blot shown is representative of 6 experiments with similar results. $I / R$ - ischemia reperfusion, VDR - vitamin $D$ receptor.

citol could lead to down-regulation of HMGB1/ TLR4/NF-KB signaling and could consequently attenuate the development and progression of hepatic I/R injury. Our results demonstrated that the plasma ALT levels showed a significant increase in the Saline + I/R group, and this elevation of the plasma ALT levels was significantly decreased by pretreatment with paricalcitol (Figure 1). These results were consistent with our histopathological findings (Figure 2). In addition, our results showed that pretreatment with paricalcitol led to a significant decrease of pro-inflammatory cytokines, such as IL- $1 \beta$, TNF- $\alpha$, and MIF in both the plasma and ischemic liver tissue of the Paricalcitol + I/R group compared to the Saline + Sham group (Figure 3). These findings were consistent with recently published results on the effect of vitamin $D$ in hepatic I/R injury [18]. Although little has been elucidated regarding the mechanisms underlying the protective effect of vitamin $D$ in I/R injury, a recent study conducted in a renal I/R model showed that pretreatment with paricalcitol could ameliorate inflammation and damage by blocking activation of the TLR4-NF-kB pathway [17]. Herein, we demon- strated, for the first time, that pretreatment with paricalcitol attenuates $\mathrm{I} / \mathrm{R}$ injury by modulating TLR4/NF-KB signaling in the liver (Figures 4 and 5).

The VDR was recognized as a member of a superfamily of nuclear receptors. This receptor with its ligand in target cells mediates biological effects, thereby regulating the transcription of target genes (the genomic pathway in non-classical tissues) [28]. Interestingly, VDRs are present not only in tissues participating in the classic actions of vitamin $\mathrm{D}$ (such as bone, skin, gut, and kidneys) but also in non-classical tissues (such as brain, heart, immune cells, liver, muscle, and adipose tissue) [22]. Many of these non-classical tissues also contain vitamin D-activating enzymes wherein non-classical actions occur via local activation of vitamin $D$ [29]. With regard to the implications of the VDR for TLR-mediated innate immunity, early evidence has demonstrated that TLR activation of human macrophages results in increased expression of the VDR and VDR target genes and leads to the induction of an antimicrobial peptide (cathelicidin) and the killing of intracellular M. tuberculosis [26]. Moreover, Gambhir et al. reported 
that the influence of vitamin D3 on TLR4-induced activation of antigen-presenting cells is dependent on the order of the VDR and TLR4 engagement in vitro [30]. To investigate potential interactions between the VDR and TLR4, we examined whether VDR expression could be up-regulated via activated TLR4 signaling after hepatic I/R injury. The most important observation of the present study is that the expression of the VDR was up-regulated in liver tissues from the Saline $+I / R$ group (Figure 6). Strikingly, the interesting point is the observation of down-regulation of the VDR expression response in parallel with the modulation of TLR4 signaling by pretreatment with paricalcitol. These findings are contrary to a previous study in a murine model of renal I/R that demonstrated that I/R caused significant suppression of VDR expression. On the other hand, paricalcitol pretreatment led to increased expression of VDR [16]. It is believed that this conflict of the results between the hepatic and renal I/R rat models may be due to the tissue-specific action of VDR. Our data showed that the expression of the VDR was paralleled by the patterns of TNF- $\alpha$, HMGB1, TLR4, and NF- $\kappa B$ expression in the liver tissue of each group. These results raise the possibility that vitamin D down-regulates the TLR4/NF- $\mathrm{B}$ signaling pathway via genomic pathways (non-classical actions) in the liver. Future studies are warranted to investigate the roles of vitamin $D$ and the VDR for controlling I/R injury in the liver.

Our study had some limitations. First, we did not confirm the intracellular localization of VDR by confocal immunofluorescence imaging. Confocal immunofluorescence microscopy may be useful for identifying the role of the VDR. Second, we detected that the pro-inflammatory cytokines of the plasma and liver tissue were increased in the Paricalcitol + Sham group compared to the Saline + Sham group in this study, even though $20 \mu \mathrm{g} /$ $\mathrm{kg}$ of paricalcitol has no influence on plasma ALT and calcium levels. We suggest that the high dose of paricalcitol $(20 \mu \mathrm{g} / \mathrm{kg})$ itself used in the present study causes the production of the pro-inflammatory cytokines as an inflammagen of the liver. Finally, this study was conducted in rats, and the ability to translate these findings to humans is currently unclear.

In conclusion, our study demonstrated that pretreatment with vitamin D (paricalcitol, $20 \mu \mathrm{g}$ / $\mathrm{kg}$ ip) attenuates hepatic I/R injury in rats. We also presented a mechanism by which paricalcitol down-regulates the TLR4 signaling pathway in an in vivo model of hepatic I/R. Consequently, the results provide some evidence that the vitamin $D$ analogue paricalcitol could be a potential nutritional therapeutic agent against hepatic I/R injury.

\section{Acknowledgments}

Min Sung Kim and Soyoung Lee contributed equally to this work.

This research was supported by the Eulji MediBio Research Institute Grant 2013-SN0003 from the Eulji University School of Medicine in Korea.

\section{Conflict of interest}

The authors declare no conflict of interest.

\section{References}

1. Tsung A, Hoffman RA, Izuishi K, et al. Hepatic ischemia/ reperfusion injury involves functional TLR4 signaling in nonparenchymal cells. J Immunol 2005; 175: 7661-8.

2. Lemasters JJ, Thurman RG. Reperfusion injury after liver preservation for transplantation. Annu Rev Pharmacol Toxicol 1997; 37: 327-38.

3. Sugawara Y, Kubota K, Ogura T, et al. Increased nitric oxide production in the liver in the perioperative period of partial hepatectomy with Pringle's maneuver. J Hepatol 1998; 28: 212-20.

4. Trocha M, Merwid-Lad A, Chlebda E, et al. Influence of ezetimibe on selected parameters of oxidative stress in rat liver subjected to ischemia/reperfusion. Arch Med Sci 2014; 10: 817-24.

5. Fondevila C, Busuttil RW, Kupiec-Weglinski JW. Hepatic ischemia/reperfusion injury: a fresh look. Exp Mol Pathol 2003; 74: 86-93.

6. Wanner GA, Ertel W, Muller P, et al. Liver ischemia and reperfusion induces a systemic inflammatory response through Kupffer cell activation. Shock 1996; 5: 34-40.

7. Kaczorowski DJ, Nakao A, Mollen KP, et al. Toll-like receptor 4 mediates the early inflammatory response after cold ischemia/reperfusion. Transplantation 2007; 84: 1279-87.

8. Tsung $A$, Klune JR, Zhang $X$, et al. HMGB1 release induced by liver ischemia involves Toll-like receptor 4-dependent reactive oxygen species production and calcium-mediated signaling. J Exp Med 2007; 204: 2913-23.

9. Zhai Y, Shen XD, O'Connell R, et al. Cutting edge: TLR4 activation mediates liver ischemia/reperfusion inflammatory response via IFN regulatory factor 3-dependent MyD88-independent pathway. J Immunol 2004; 173: 7115-9.

10. DeLuca HF. Overview of general physiologic features and functions of vitamin D. Am J Clin Nutr 2004; 80: 1689s-96s.

11. Horiuchi H, Nagata I, Komoriya K. Protective effect of vitamin D3 analogues on endotoxin shock in mice. Agents Actions 1991; 33: 343-8.

12. Moller S, Laigaard F, Olgaard K, Hemmingsen C. Effect of 1,25-dihydroxy-vitamin D3 in experimental sepsis. Int J Med Sci 2007; 4: 190-5.

13. Ekici F, Ozyurt B, Erdogan H. The combination of vitamin D3 and dehydroascorbic acid administration attenuates brain damage in focal ischemia. Neurol Sci 2009; 30: 207-12.

14. Xiang G, Seki T, Schuster MD, et al. Catalytic degradation of vitamin D up-regulated protein 1 mRNA enhances cardiomyocyte survival and prevents left ventricular remodeling after myocardial ischemia. J Biol Chem 2005; 280: 39394-402.

15. Azak A, Huddam B, Haberal N, et al. Effect of novel vitamin $D$ receptor activator paricalcitol on renal ischaemia/ 
reperfusion injury in rats. Ann R Coll Surg Engl 2013; 95: 489-94.

16. Hwang HS, Yang KJ, Park KC, et al. Pretreatment with paricalcitol attenuates inflammation in ischemia-reperfusion injury via the up-regulation of cyclooxygenase-2 and prostaglandin E2. Nephrol Dial Transplant 2013; 28: 1156-66.

17. Lee JW, Kim SC, Ko YS, et al. Renoprotective effect of paricalcitol via a modulation of the TLR4-NF-kappaB pathway in ischemia/reperfusion-induced acute kidney injury. Biochem Biophys Res Commun 2014; 444: 121-7.

18. Seif AA, Abdelwahed DM. Vitamin D ameliorates hepatic ischemic/reperfusion injury in rats. J Physiol Biochem 2014; 70: 659-66.

19. Kim K, Jung N, Lee K, et al. Dietary omega-3 polyunsaturated fatty acids attenuate hepatic ischemia/reperfusion injury in rats by modulating toll-like receptor recruitment into lipid rafts. Clin Nutr 2013; 32: 855-62.

20. Gill R, Tsung A, Billiar T. Linking oxidative stress to inflammation: Toll-like receptors. Free Radic Biol Med 2010; 48: 1121-32.

21. Poltorak A, He X, Smirnova I, et al. Defective LPS signaling in $\mathrm{C} 3 \mathrm{H} / \mathrm{HeJ}$ and $\mathrm{C} 57 \mathrm{BL} / 10 \mathrm{ScCr}$ mice: mutations in Tlr4 gene. Science 1998; 282: 2085-8.

22. Bikle D. Nonclassic actions of vitamin D. J Clin Endocrinol Metab 2009; 94: 26-34.

23. Manolagas SC, Haussler MR, Deftos LJ. 1,25-dihydroxyvitamin D3 receptors in cancer. Lancet 1980; 1: 828.

24. Abe E, Miyaura C, Sakagami H, et al. Differentiation of mouse myeloid leukemia cells induced by 1 alpha,25-dihydroxyvitamin D3. Proc Natl Acad Sci U S A 1981; 78: 4990-4.

25. Abe E, Miyaura C, Tanaka H, et al. 1 alpha,25-dihydroxyvitamin D3 promotes fusion of mouse alveolar macrophages both by a direct mechanism and by a spleen cell-mediated indirect mechanism. Proc Natl Acad Sci U S A 1983; 80: 5583-7.

26. Liu PT, Stenger S, Li H, et al. Toll-like receptor triggering of a vitamin D-mediated human antimicrobial response. Science 2006; 311: 1770-3.

27. Sadeghi K, Wessner B, Laggner $U$, et al. Vitamin D3 down-regulates monocyte TLR expression and triggers hyporesponsiveness to pathogen-associated molecular patterns. Eur J Immunol 2006; 36: 361-70.

28. Segura C, Alonso M, Fraga C, Garcia-Caballero T, Dieguez C, Perez-Fernandez R. Vitamin D receptor ontogenesis in rat liver. Histochem Cell Biol 1999; 112: 163-7.

29. Baeke F, Takiishi T, Korf H, Gysemans C, Mathieu C. Vitamin D: modulator of the immune system. Curr Opin Pharmacol 2010; 10: 482-96.

30. Gambhir V, Kim J, Siddiqui S, et al. Influence of 1,25-dihydroxy vitamin D3 on TLR4-induced activation of antigen presenting cells is dependent on the order of receptor engagement. Immunobiology 2011; 216: 988-96. 